\title{
Fast-discharge excitation of hot capillary plasmas for soft-x-ray amplifiers
}

\author{
J. J. Rocca, O. D. Cortázar, B. Szapiro, K. Floyd, and F. G. Tomasel \\ Department of Electrical Engineering, Colorado State University, Fort Collins, Colorado 80523
}

(Received 19 August 1992)

\begin{abstract}
High-temperature $\left(T_{e}>150 \mathrm{eV}\right)$, small-diameter $(\sim 200 \mu \mathrm{m})$ plasma columns have been efficiently generated by very fast ( $13 \mathrm{~ns}$ rise time, $28 \mathrm{~ns}$ full width at half maximum) pulsed discharge excitation of capillary channels filled with preionized gas. Discharges in argon-filled capillaries at currents between 20 and $60 \mathrm{kA}$ produced plasmas with Ar X-Ar XIV line emission, in which the degree of ionization was controlled by the magnitude of the current pulse. The characteristics of these plasmas differ from those created by vacuum discharges in the same capillaries and approach those necessary for soft-x-ray amplification in low- $Z$ elements.
\end{abstract}

PACS number(s): 52.80. $-\mathrm{s}, 42.55 . \mathrm{Vc}$

Herein we report the efficient generation of hot $\left(T_{e}>150 \mathrm{eV}\right)$ plasma columns of small diameter $(\sim 200$ $\mu \mathrm{m})$ by fast current pulse excitation of capillary channels filled with preionized gas. These plasmas are of smaller diameter and hotter than those observed in evacuated capillaries excited by a similar current pulse. Soft-x-ray spectra from argon plasmas created by these relatively compact capillary discharges at currents $I<60 \mathrm{kA}$ are similar to those of plasmas generated by $\geq 1$ MA current implosions in large pulsed power machines. This efficient generation of highly ionized capillary plasma columns can potentially impact the development of small-scale soft-x-ray lasers.

Capillary discharges have been initially studied as sources of soft-x-ray radiation for spectroscopy, x-ray lithography, microscopy [1-3]. More recently these discharges have been proposed as gain medium for compact, high-efficiency soft-x-ray lasers [4]. Recombination and collisional excitation laser schemes both require the generation of hot, highly ionized plasma columns with a large optical length in order to achieve a significant amplification. The recombination scheme, in addition, requires rapid cooling of the plasma. A small plasma diameter is also crucial in both cases to avoid quenching of the population inversion by trapping of resonant radiation.

The possibility of obtaining soft-x-ray amplification by plasma recombination in a capillary discharge has recently motivated several experimental studies of the soft-xray emission from evacuated polyacetal and polyethylene capillaries $0.5-1 \mathrm{~mm}$ in diameter [5-8]. Spectra from these experiments are consistent with plasma temperatures in the range of $15-70 \mathrm{eV}$. In evacuated capillaries the discharge starts by surface flashover on the capillary walls, and the plasma column is composed of material ablated from the capillary walls. In discharges such as those in the experiments mentioned above, in which the rise time of the current pulse was $\geq 50 \mathrm{~ns}$, the plasma remains coupled to the walls during the course of the current pulse. The material injected into the plasma by wall ablation and the electron heat conduction from the plasma column to the walls limit to relatively low values the temperature that can be achieved. An increase of the excitation current does not necessarily result in a significantly higher plasma temperature, as an increase in the discharge energy results in more ablated material and in a higher plasma density. In effect, high current pulses $(500 \mathrm{kA})$ of $300-\mathrm{ns}$ rise time injected through narrow capillary channels have created cold $\left(T_{e}=10 \mathrm{eV}\right)$, highdensity plasmas which have been used for the study of transport coefficients in partially degenerate, strongly coupled plasmas [9].

Higher temperatures in evacuated capillaries can result from utilizing a very fast rise of the current pulse to limit the amount of material ablated from the capillary walls before the magnetic field of the current pulse compresses the plasma detaching it from the walls. High-temperature plasmas, with emission from F VIII and F IX have been reported to result from the excitation of 1-mm diameter evacuated teflon capillaries with 120-kA current pulses having a fast $(\leq 10 \mathrm{~ns})$ rise time [10]. Experiments we have conducted in teflon capillaries of the same diameter excited by 11-ns rise-time current pulses of up to $86 \mathrm{kA}$ have produced colder plasmas, with emission from F VII and F VIII. The different degree of ionization observed in the two experiments is probably not caused by the difference in peak currents, but more likely by the specific shape of the current pulse, which determines the amount of mass ablated at the onset of the discharge. The characteristics of the plasmas generated in evacuated capillary discharges depend on this and other conditions, such as the uniformity of the breakdown, which are difficult to control.

In the experiments reported herein hot plasma columns of diameter much smaller than the capillary diameter are generated by tailoring the conditions at the initiation of the discharge to allow for a rapid detachment of the plasma from the capillary walls. A fast rising current pulse encounters a selected low mass density of uniformly preionized material filling the capillary. The rapid compression that results produces a highly ionized plasma column which is observed to be of smaller diameter and hotter than those produced by a similar current pulse in evacuated capillaries of the same size.

The development of these discharges in capillaries 
filled with the preionized gas resembles the formation of a compressional $z$-pinch by a rapidly rising current pulse [11-14]. However, in the capillaries the initial diameter of the plasma is nearly an order of magnitude smaller than those commonly used in low density $z$-pinch experiments, and hot plasmas with small diameters are produced utilizing only modest discharge energies. The argon spectra reported herein for currents $I<60 \mathrm{kA}$ resemble those of argon plasmas produced by mega-ampere driving currents in the Gamble II and Python multiterawatt pulse generators $[12,15]$.

The experiments were conducted utilizing a recently developed high-voltage fast pulse generator which produces current pulses having a $10 \%$ to $90 \%$ rise time of 13 $\mathrm{ns}$ and a full width at half maximum (FWHM) of $28 \mathrm{~ns}$. The pulse generator and capillary discharge set up are schematically illustrated in Fig. 1. The capillary is in the axis of a 3-nF circular parallel plates capacitor containing ethylene glycol as dielectric. The capacitor was charged by a seven-stage, 700-kV Marx generator. The capillaries were excited by discharging this capacitor through a low inductance circuit which includes the capillary and a spark gap switch controlled with $\mathrm{SF}_{6}$. The current pulse was monitored with a Rogowski coil having a response time of less than 2 ns. To conduct the experiments reported herein the pulse generator was designed to also

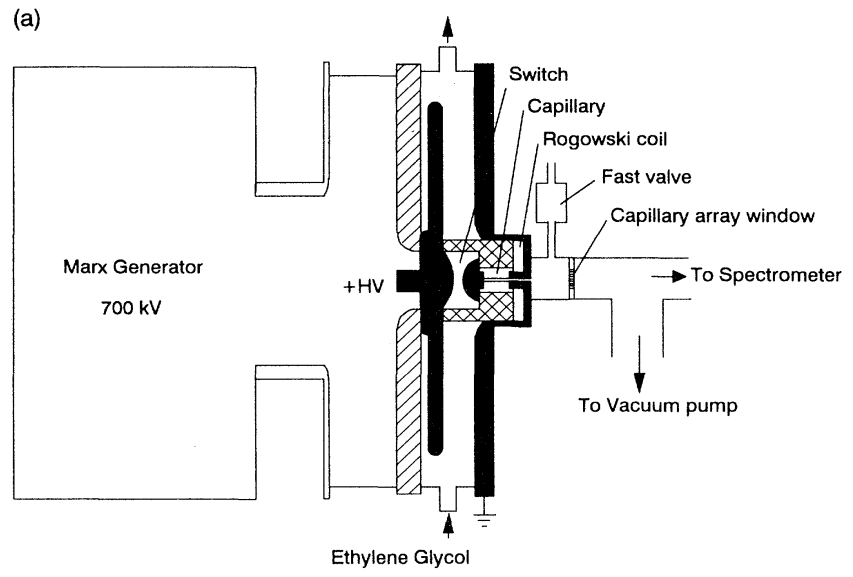

(b)

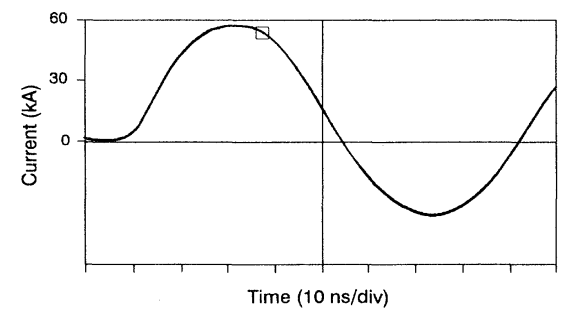

FIG. 1. (a) Schematic illustration of the fast capillary discharge setup. (b) Typical current pulse corresponding to a discharge through a $2.5-\mathrm{mm}$-diameter, 1-cm-long argon-filled capillary. The mark identifies the time at which the spectra of Figs. 2 and 3 were obtained. produce a preionization pulse with a current of 20-40 A and a duration of approximately $2 \mu$ s immediately preceding the fast discharge pulse. The studies were conducted in polyacetal and in teflon capillary channels having either 1 or $5 \mathrm{~cm}$ in length and diameters of 1.5 and $2.5 \mathrm{~mm}$. The capillaries were evacuated to a pressure below $2 \times 10^{-5}$ Torr and argon gas was injected shortly before the initiation of the current pulse utilizing a fast valve. Several hundred shots were fired in a single 2.5$\mathrm{mm}$ polyacetal capillary without observing any significant sign of deterioration of the capillary structure.

The axial soft-x-ray emission from the plasma was observed through a hole in the anode electrode. The radiation from the plasma was collected and focused in the slit of a 1-m grazing incidence vacuum spectrograph by a gold-coated cylindrical mirror positioned at $86^{\circ}$ with respect to the incoming radiation. The spectrograph contained a gold-coated grating ruled at 1200 lines per millimeter with a blaze angle of $1^{\circ}$. The dispersed radiation was detected by a windowless multichannel plate intensified diode array which was gated to obtain spectra with a temporal resolution of approximately $5 \mathrm{~ns}$.

Figures 2(a)-2(c) show time-resolved soft-x-ray spectra covering the spectral range between 150 and $193 \AA$ from a $2.5-\mathrm{mm}$ diameter, 1-cm-long polyacetal capillary filled with approximately $100 \mathrm{~Pa}$ of preionized argon gas for three values of the discharge current. All the spectra shown in this figure were obtained at the time indicated in Fig. 1(b), 6 ns after the peak of the current pulse. Comparison of spectra corresponding to the different currents shows that it is possible to select the degree of ionization by varying the amplitude of the current pulse. The spectrum in Fig. 2(a), which corresponds to a $23-\mathrm{kA}$ current pulse, is dominated by line emission from fluorine-like argon ArX. Emission from Ar XI lines and from $\mathrm{O}$ vi lines are also observed. The spectrum of 43kA discharge, shown in Fig. 2(b), corresponds to a higher degree of ionization. At this current the Ar XI transitions are more intense and emission from Ar XII lines is also observed. Similar spectra were observed from discharges in 5-cm-long capillaries of the same diameter. The features of the 43-kA spectrum are remarkably similar to those reported for an argon $z$-pinch implosion driven by a 1-MA current pulse from the Gamble II generator (see Fig. 6 in Ref. [12]). Figure 2(c) shows that further increase of the discharge current to $56 \mathrm{kA}$ caused the appearance of Ar XIII transitions and a decrease in the abundance of Ar X.

A calculation of the ionization times of argon utilizing the ionization coefficients of Lotz [16] for the value of the electron density given below indicates that the electron temperature must be higher than $120 \mathrm{eV}$ for Ar XIII lines to be observed $30 \mathrm{~ns}$ after the beginning of the discharge current pulse, as shown by the spectrum of Fig. 2(c). The electron density was estimated from the ratio of intensities of the 197.95 and $171.86 \AA$ lines of Ar XI, which is sensitive to the electron density in the range from $1 \times 10^{17}$ $\mathrm{cm}^{-3}$ to about $1 \times 10^{19} \mathrm{~cm}^{-3}$ [17]. For a 55-kA discharge in argon at about $100 \mathrm{~Pa}$ this intensity ratio was measured to be 0.6 , corresponding to an electron density of about $5 \times 10^{18} \mathrm{~cm}^{-3}$ in the region of the plasma where the 
Ar XI emission occurs, based on computations for a plas$\mathrm{ma}$ at $170 \mathrm{eV}$ [17].

A higher degree of ionization corresponding to a hotter plasma was observed in the argon discharges conducted in a $1.5-\mathrm{mm}$ capillary. The time-resolved spectra of Fig. 3 , which corresponds to a 51-kA discharge current pulse in that capillary, shows the emission from Ar XIV lines. Strong transitions corresponding to Ar XIII are also observed, while the emission from Ar XII lines is weak, an indication that the population of the ion nears complete ionization. Calculations of the ionization times [16] show that in this discharge the electron temperature surpasses $150 \mathrm{eV}$. These temperatures are higher than those observed in evacuated capillaries excited by a similar current pulse. The spectra from discharges in evacuated polyacetal capillaries 1.5 and $2.5 \mathrm{~mm}$ in diameter show that the O VI $4 d-2 p$ and the O VII $3 d-2 p$ lines have similar intensities, an indication that the plasma temperature remained under $90 \mathrm{eV}$. As discussed below, time- resolved soft-x-ray pinhole images show a significant difference in the spatial distribution of the plasmas reported herein and those created in the same capillaries by similar current pulses under vacuum.

End-on soft-x-ray images of the capillary plasmas were obtained utilizing a pinhole camera consisting of a 90$\mu$ m-diam pinhole placed at $38 \mathrm{~cm}$ from the capillary, and a vacuum soft- $x$-ray image intensifier consisting of a multichannel plate (MCP) and a phosphorous screen. The camera has a calculated magnification of 3 , which was verified experimentally by recording the extent of the emission from a direct current low-pressure helium capillary lamp $1 \mathrm{~mm}$ in diameter placed for the purpose of this calibration at the location of the capillary. The spectral sensitivity of the imaging camera was limited to wavelengths below approximately $350 \AA$ by a 100-nm-thick carbon foil filter placed over the pinhole and by the photoelectric emission response of the $\mathrm{MgO}$ coating utilized on the MCP. Two-dimensional images of the plasma
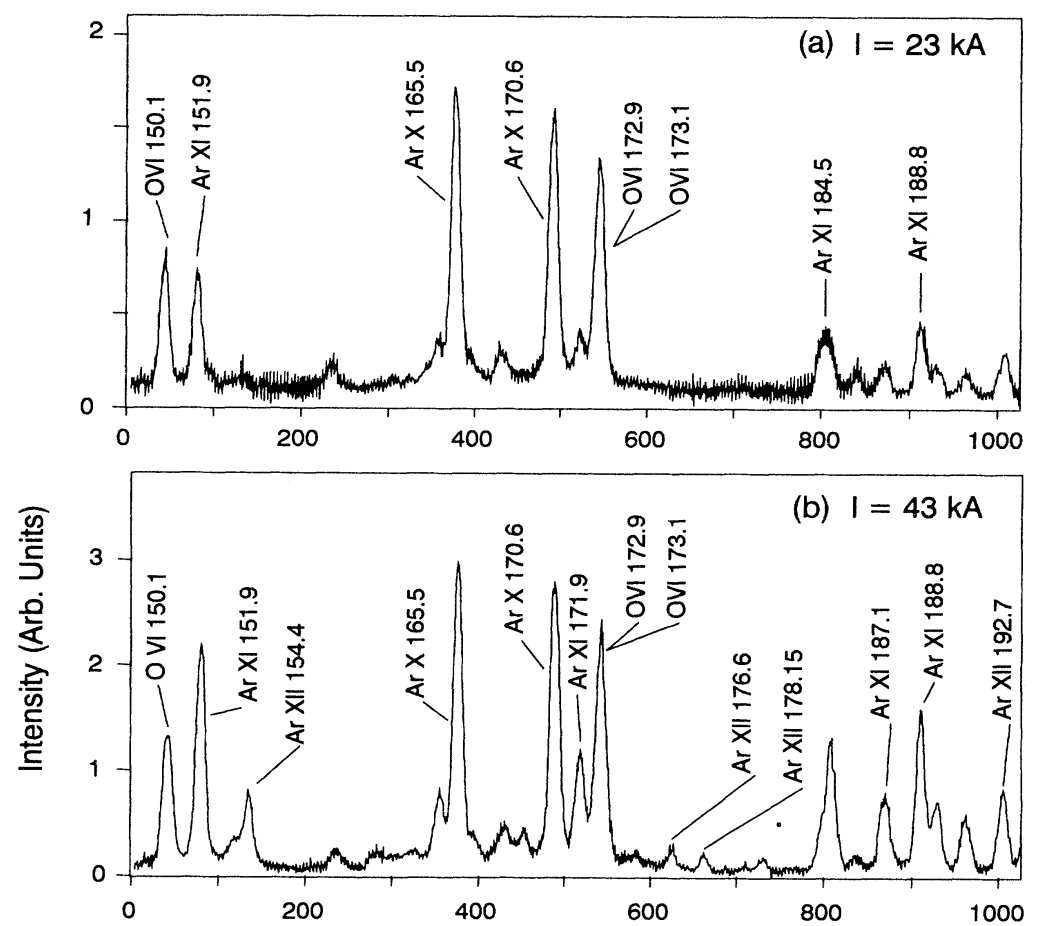

FIG. 2. Time-resolved spectra of the soft-xray emission (150-193 $\AA$ ) from a discharge through a 2.5 -mm-diam capillary filled with approximately $100 \mathrm{~Pa}$ of argon for three values of the excitation current. The spectra have a temporal resolution of $5 \mathrm{~ns}$ and were obtained $6 \mathrm{~ns}$ after the peak of the current pulse.

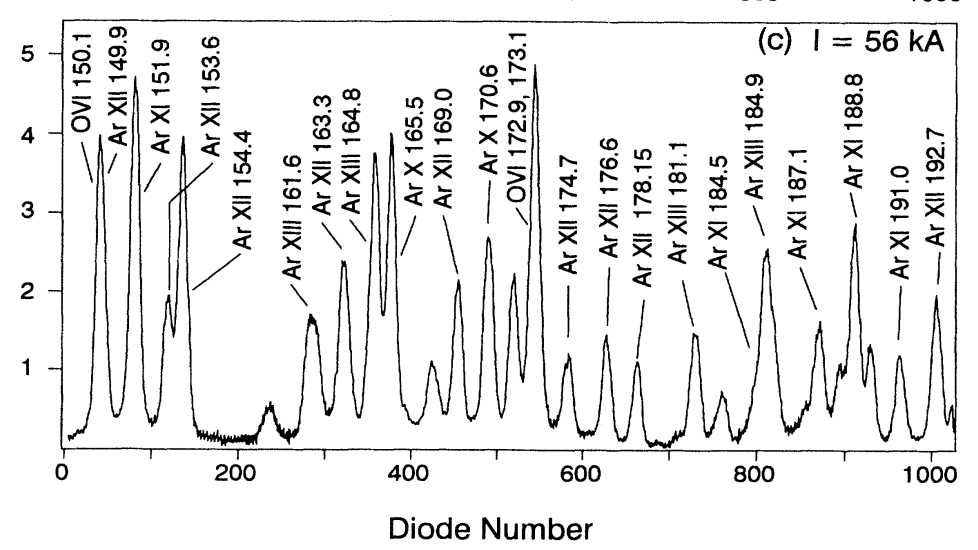




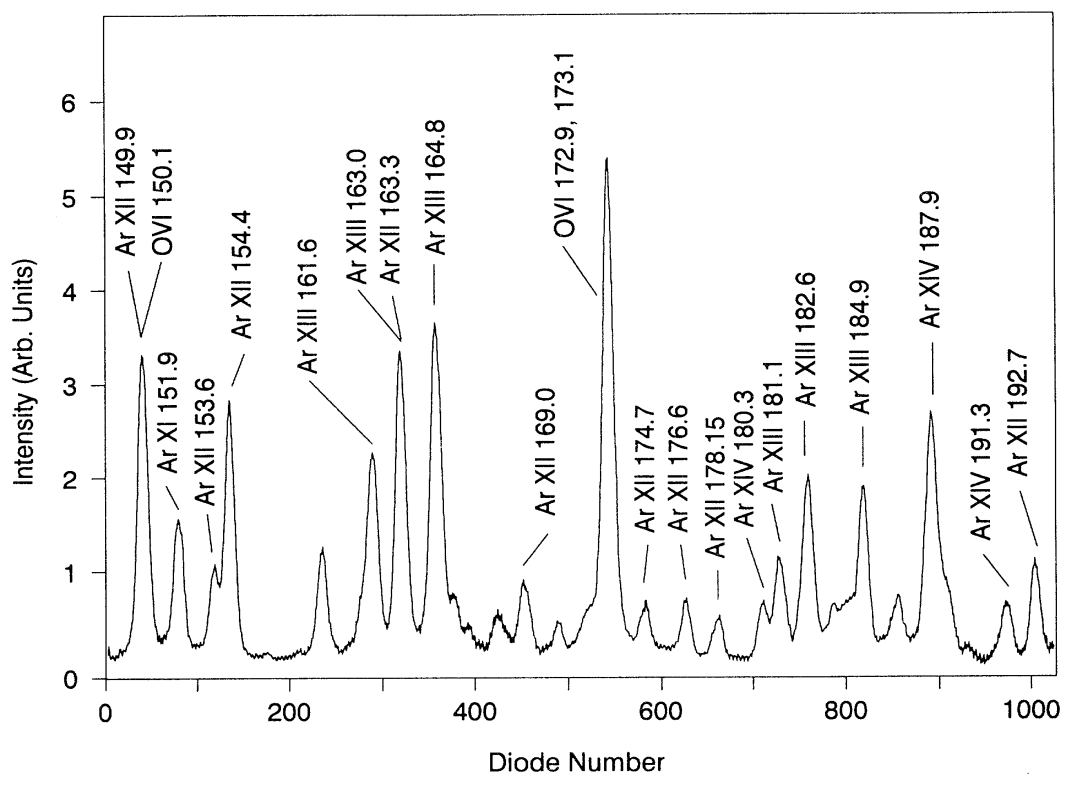

FIG. 3. Time-resolved spectrum of the soft-x-ray emission (150-193 $\AA$ ) from a 1.5 mm-diam capillary excited by a 51-kA current pulse. The spectra was obtained at the time indicated in Fig. 1, 6 ns after the peak of the current pulse. were recorded in photographic film. The radial intensity profile of the soft-x-ray image was also recorded directly from the phosphorous screen utilizing a linear diode array detector. In both cases a temporal resolution of approximately $7 \mathrm{~ns}$ was obtained by gating the gain of the MCP intensifier.

Figure 4 compares the measured time-integrated soft$\mathrm{x}$-ray radial intensity profile from a 44-kA discharge in a 2.5-mm-diam capillary filled with preionized argon to that corresponding to a vacuum discharge in the same capillary. In the first case the plasma that emits the soft $x$ rays occupies the central region of the capillary and has a FWHM of approximately $0.26 \mathrm{~mm}$. In contrast, the soft-x-ray emission from the evacuated capillary is less intense and originates from a significantly broader plasma column, having a FWHM diameter of approximately 1.1 $\mathrm{mm}$.

Time-resolved pinhole images describing the evolution of the argon plasma column are shown in Fig. 5. All of the soft-x-ray pinhole images obtained during the first half cycle of the current pulse are highly reproducible, axially symmetric, and smooth, an indication that the plasma column is well behaved and free of instabilities. The fast current pulse rapidly detaches the plasma column from the capillary walls and collapse of the plasma occurs before the earliest time at which we could obtain a time-resolved pinhole image, $8 \mathrm{~ns}$ after the peak of the current pulse. At this time, the FWHM diameter of the soft-x-ray emitting region of the plasma column is measured to be approximately $0.19 \mathrm{~mm}$. Thereafter, the diameter of the plasma column is observed to expand continuously and without disruptions to reach a diameter of $0.42 \mathrm{~mm}$ by the end of the first half cycle of the current pulse. The expansion continues during the first part of the second half cycle of the current pulse. At the time of the end of the first half cycle of the current the plasma is measured to expand at a velocity of approximately
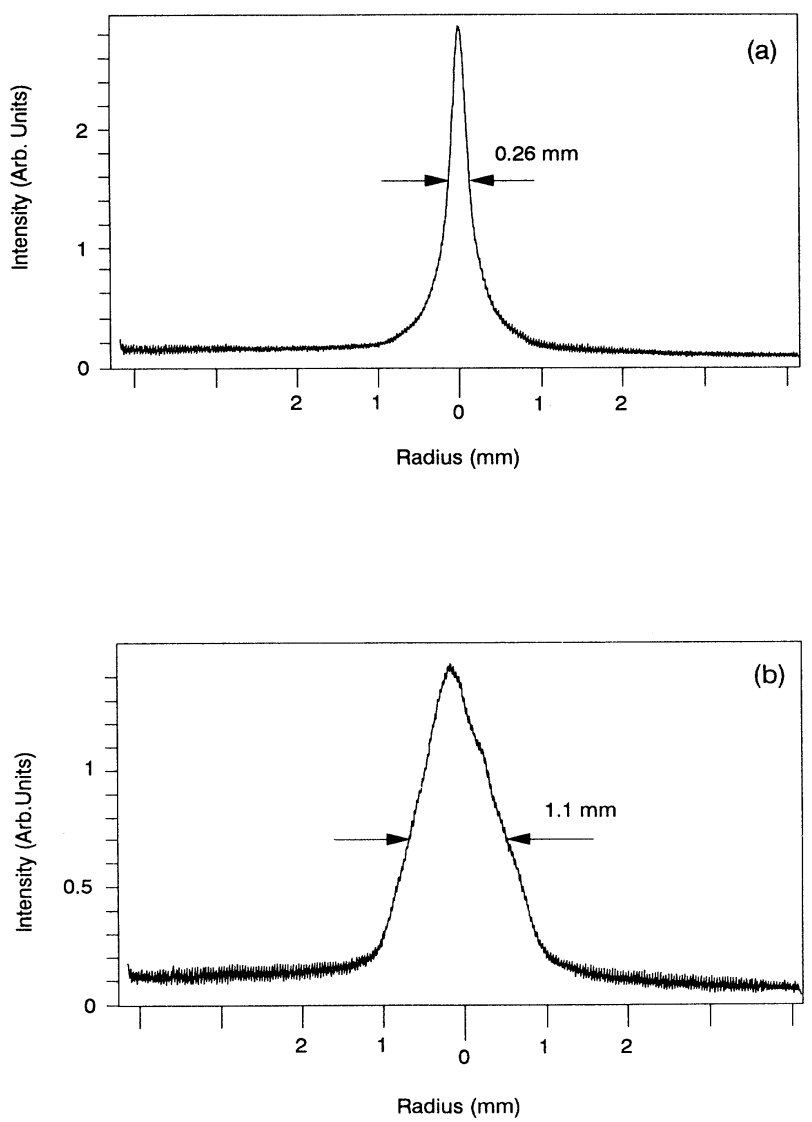

FIG. 4. Radial intensity profiles of time-integrated pinhole camera images of the axial soft-x-ray emission from a $2.5-\mathrm{mm}$ diam capillary excited by a 44-kA current pulse for (a) capillary filled with approximately $100 \mathrm{~Pa}$ of preionized argon gas, and (b) evacuated capillary. 
$1.5 \times 10^{7} \mathrm{~cm} / \mathrm{sec}$. The rapid expansion phase can be of interest for the population inversions by collisional recombination. As described below, the short-lived, small-diameter hot capillary plasmas reported herein have characteristics that approach those necessary for amplification of soft-x-ray radiation by collisional excitation of low- $Z$ ions of the Ne-like and Ni-like sequences.

The Ne-like sequence has been very successfully utilized in laser-produced plasmas to generate soft-x-ray lasing from electron-collisional excitation pumping of $3 p-3 s$
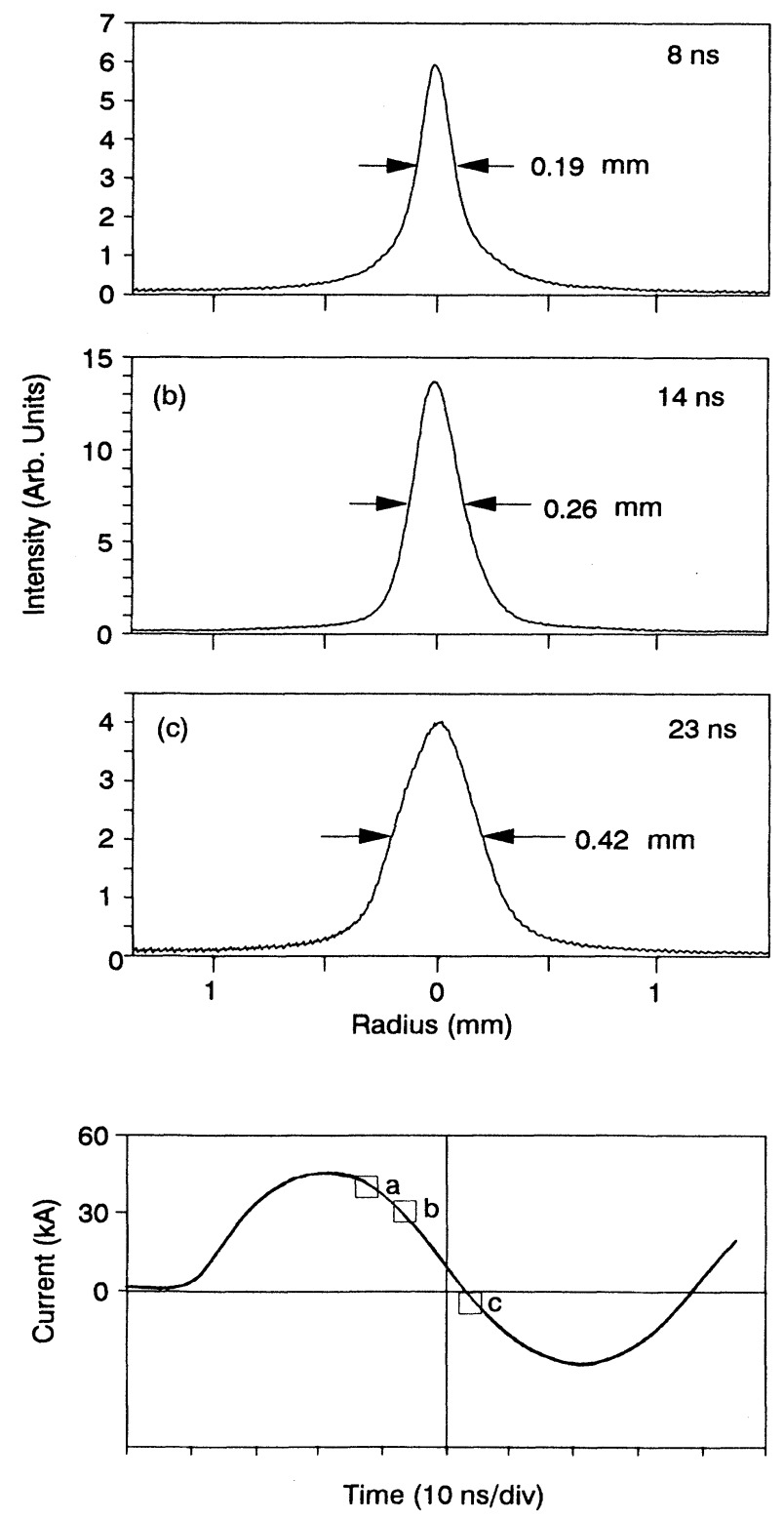

FIG. 5. Radial intensity profiles from a time-resolved sequence of on-axis soft-x-ray pinhole images from an argon capillary discharge plasma. The discharge conditions are the same than those of Fig. 4(a). The current pulse at the bottom of the figure illustrates the timing of each pinhole image. The times indicated are measured relative to the peak of the current pulse. transitions in elements with $Z$ as low as 22 (titanium) [18-21]. The favorable scaling to low $Z$ of the also successful Ni-like sequence has been predicted theoretically by Hagelstein [22]. The latter ions have the advantage of requiring reduced excitation energy for lasing in a given wavelength range, and are probably the most promising laser candidates for the capillary plasmas described above. Ar IX and KrIX are the two gaseous elements of the Ne-like and the Ni-like sequences for which the required plasma conditions for gain fall within the range of those of the capillary discharges described herein. To explore for gain in argon, a plasma temperature of approximately $80 \mathrm{eV}$ and densities between $10^{18}$ and $10^{19} \mathrm{~cm}^{-3}$ are required. For argon, however, the predicted gains are small; a gain of $0.16 \mathrm{~cm}^{-1}$ has been predicted for the $J=2-1$ Ar IX lines [23]. While a gain of $2 \mathrm{~cm}^{-1}$ has been computed for the $\left({ }^{1} S_{0^{-}}{ }^{1} P_{1}\right), 468.9-\AA$ line of Ar IX [24], this value is probably optimistic, as the difficulty in modeling the $J=0-1$ transition in Ne-like ions has frequently resulted in an overestimate of the gain as compared with the experiments [25]. To estimate the gain in the $4 d-4 p$ $\left({ }^{1} S_{0^{-}}{ }^{1} P_{1}\right), 337-\AA$ transition of Ni-like $\mathrm{Kr}$ IX, we utilized the three-level model developed by Hagelstein [22]. A predicted gain of $1.4 \mathrm{~cm}^{-1}$ results from assuming a plasma with an optimum electron density of $7.1 \times 10^{17} \mathrm{~cm}^{-3}$ and an electron temperature of $100 \mathrm{eV}$, in the case in which $50 \%$ of the ions are in the Ni-like ground state [26]. Moreover, the plasma conditions necessary for optimum gain in heavier elements of these sequences, in which the gain are predicted to be significantly larger, appear to be within the reach of this type of discharge. Plasma temperatures of approximately 150 and $200 \mathrm{eV}$ are required for maximum gain in the $3 p-3 s$ transition of Ne-like calcium and titanium [25], while also a temperature of approximately $200 \mathrm{eV}$ and an electron density of $1 \times 10^{19} \mathrm{~cm}^{-3}$ are required for Ni-like molybdenum [22]. To create a plasma with the necessary component of these nongaseous elements, capillaries containing compounds of the selected material (e.g., $\mathrm{CaH}_{2}$ in the case of a calcium plasma) can be utilized. A discharge prepulse, tailored to ablate a controlled amount of mass from the capillary walls, can be employed to create a preionized plasma column prior to the excitation with the fast current pulse. We expect that a single capillary will last a relatively large number of shots, as was the case in the experiments described herein. In this way, the repetitive generation of plasmas from nongaseous elements should be possible.

In summary, we have demonstrated the efficient generation of hot, dense capillary plasmas with a diameter of $\sim 200 \mu \mathrm{m}$ and aspect ratios up to $250: 1$ by fast discharge excitation of a preionized capillary column. The fundamental elements in the developed of these well-behaved hot plasma columns are a small-diameter capillary structure initially filled with a low-mass density plasma, and a very fast rise-time excitation pulse which rapidly detaches the plasma from the capillary walls. These capillary plasmas have characteristics that approach those necessary for soft-x-ray amplification in low- $Z$ elements, and consequently are of interest for the development of small-scale soft-x-ray amplifiers. 
We thank Dr. Peter Hagelstein for valuable suggestions and encouraging discussions related to the excitation of laser transitions in $\mathrm{Ni}$-like ions. We are also thankful to V. N. Shlyaptsev for useful discussions about collisionally excited laser schemes and to F. C. Young for making available spectral data from the argon experiments conducted at NRL with the Gamble II generator.
We acknowledge the collaboration of M. Marconi and K. Richardson in the development of the pulse generator. This research was supported by the U.S. Department of Energy, Advanced Energy Projects, Grant No. DEFG02-91ER 12110 and by the National Science Foundation, Grant No. ECS 9013372.
[1] H. Conrads, Z. Phys. 444, 200 (1967).

[2] P. Bogen, H. Conrads, G. Gatti, and W. Kohlhaas, J. Opt. Soc. Am. 58, 203 (1968).

[3] R. A. McCorkle, Appl. Phys. A 26, 261 (1981).

[4] J. J. Rocca, D. C. Beethe, and M. C. Marconi, Opt. Lett. 13, 565 (1988).

[5] C. Steden and H.-J. Kunze, Phys. Lett. A 151, 1534 (1990).

[6] C. A. Morgan, J. C. Moreno, H. R. Griem, and E. J. Iglesias, Bull. Am. Phys. Soc. 36, 2432 (1991).

[7] J. J. Rocca, M. C. Marconi, and F. G. Tomasel, IEEE J. Quantum Electron. (to be published).

[8] J. J. Rocca, M. C. Marconi, B. T. Szapiro, and J. Meyer, Proc. SPIE 1551, 275 (1991).

[9] R. L. Shepard, D. R. Kania, and L. A. Jones, Phys. Rev. Lett. 61, 1278 (1988).

[10] S. M. Zakharov, A. A. Kolomenskii, S. A. Pikuz, and A. I. Samokhin, Pis'ma Zh. Tekh. Fiz. 6, 1135 (1980) [Sov. Tech. Phys. Lett. 6, 486 (1980)].

[11] N. R. Pereira and J. Davis, J. Appl. Phys. 64, R1 (1988).

[12] P. G. Burkhalter, G. Mehlman, F. C. Young, S. J. Stephanakis, V. E. Scherrer, and D. A. Newman, J. Phys. (Paris) Colloq., Suppl. 10, 47, C-247 (1986). See argon spectra in Fig. 6.

[13] W. Hartmann, H. Bauer, J. Christiansen, K. Frank, H. Kuhn, M. Stetter, R. Tkotz, and T. Wagner, Appl. Phys. Lett. 58, 2619 (1991).

[14] J. M. Bayley, P. Baldock, and A. E. Dangor, in Dense ZPinches, edited by Nino Pereira, Jack Davis, and Norman Rostoker, Proceedings of a Conference Concerning the Fusion Applications of the Linear Z-pinch, Laguna Beach, CA, 1989, AIP Conf. Proc. No. 195 (AIP, New York, 1989), p. 481.

[15] J. D. Perez, L. F. Chase, R. E. McDonald, L. Tannenwald, and B. A. Watson, J. Appl. Phys. 52, 670 (1981).

[16] W. Lotz, Z. Phys. 216, (1968).

[17] U. Feldmann and G. A. Doschek, J. Opt. Soc. Am. 67, 726 (1977).

[18] D. L. Matthews, P. L. Hagelstein, M. D. Rosen, M. J. Eckart, N. M. Ceglio, A. U. Hazi, H. Medecki, B. J. MacGowan, J. E. Trebes, B. L. Whitten, E. M. Campbell, C. W. Hatcher, A. M. Hawryluk, R. L. Kauffman, L. D. Pleasance, G. Rambach, J. H. Scofield, G. Stone, and T. A. Weaver, Phys. Rev. Lett. 54, 110 (1985).

[19] B. J. MacGowan, M. D. Rosen, M. J. Eckart, P. L. Hagelstein, D. L. Mattlews, D. G. Nilson, T. W. Phillips, J. H. Scofield, G. Shimkaveg, J. E. Trebes, R. S. Walling, B. L. Whitten, and J. G. Woodworth, J. Appl. Phys. 61, 5423 (1983).

[20] T. N. Lee, E. A. McLean, and R. C. Elton, Phys. Rev. Lett. 39, 1185 (1987).

[21] T. Boehly, M. Russoto, R. S. Craxton, R. Epstein, B. Yaakobi, L. da Silva, J. Nilsen, E. A. Chandler, D. J. Fields, B. J. MacGowan, D. L. Matthews, J. H. Scofield, and G. Shimkaveg, Phys. Rev. A 42, 6462 (1990).

[22] P. L. Hagelstein, Proc. SPIE 1551, 254 (1991).

[23] B. Whitten and R. Walling (unpublished).

[24] U. Feldman, J. F. Seely, and G. A. Doschek, J. Phys. C6, 187 (1986).

[25] B. L. Whitten, R. A. London, and R. S. Walling, J. Opt. Soc. Am. B 5, 2537 (1988).

[26] J. J. Rocca, B. T. Szapiro, O. D. Cortázar, F. G. Tomasel, M. C. Marconi, J. Hung, and K. Floyd, paper presented at Third International Colloquium on X-ray Lasers, Schliersee, Germany, 1992, edited by E. Fill, Institute of Physics Conf. Ser. No. 125 (Institute of Physics, London, 1992), p. 427. 